\title{
Management tool to guide rabies elimination programmes
}

\author{
Kristyna Rysava ${ }^{\star 2,1}$, Tamara Mancero ${ }^{3}$, Eduardo Caldas ${ }^{4}$, Mary Carvalho ${ }^{6}$, \\ Veronica Gutierrez ${ }^{5}$, Daniel Haydon², Paul Johnson², Rebecca Mancy², \\ Jesus F. Gonzalez Roldan ${ }^{5}$, Victor D. Vilas ${ }^{6}$ and Katie Hampson ${ }^{2}$
}

${ }^{1}$ University of Warwick, School of Life Sciences, Coventry, United Kingdom; ${ }^{2}$ University of Glasgow, Institute of Biodiversity, Animal Health \& Comparative Medicine, Glasgow, United Kingdom; ${ }^{3}$ Pan American Health Organization (Mexico), Mexico City, Mexico; ${ }^{4}$ Unidade Técnica de Vigilância de Zoonoses, Brasilia, Brazil; ${ }^{5}$ CENAPRECE, Mexico City, Mexico; ${ }^{6}$ Pan American Health Organization (Brazil), Rio de Janeiro, Brazil

\section{Objective}

To provide surveillance tools to support policymakers and practitioners to identify epidemiological situations and inform the progressive implementation of rabies elimination programmes.

\section{Introduction}

Global targets for elimination of human rabies mediated by dogs have been set for 2030. In the Americas countries are progressing towards interruption of transmission and declaration of rabies freedom ${ }^{1}$. Guidance for managing elimination programmes to ensure continued progress during the endgame is critical, yet often limited and lacking in specific recommendations. Characteristic spatiotemporal incidence patterns are indicative of progress, and through their identification, tailored guidance can be provided.

\section{Methods}

Using SIRVERA, a surveillance database for rabies in the Americas $^{2}$, we developed a classification framework for identification of epidemiological situations at subnational level. Each situation exhibits a characteristic pattern identified via a set of objective criteria including trends in case detection, assessment of virus variants, case locations and measures of incursion risk.

We refined our framework through application to Mexico in consultation with stakeholders. To understand factors predicting incursions we analysed state-level data on vaccination campaigns, populations and socioeconomic indicators employing multivariate regression models.

\section{Results}

We were able to classify all states in Mexico and provide correspondingly tailored guidance. Control efforts have resulted in progress towards elimination; however rabies still circulates endemically in one state Chiapas, putting its neighbours at risk of re-emergence.

Epidemiological and socioeconomic factors associated with incursions were primarily geographic proximity to endemic and highprevalence states, and inconsistent vaccination campaigns associated with a low human development index.

\section{Conclusions}

Our management tool can support rabies programme managers at subnational levels to identify their epidemiological situation, develop tailored plans to meet targets, and sustainably maintain rabies freedom, as demonstrated for Mexico. Effective surveillance is critical for disease elimination. Control options differ depending on whether disease circulates intermittently through reintroductions or persists focally, but with poor detection these situations might be indistinguishable. Our analysis enables identification of at-risk areas and methods to reduce risk. Investment in remaining endemic areas, through improved implementation and monitoring of mass dog vaccinations, is expected to provide the most cost-effective approach to elimination whilst preventing re-emergence elsewhere.

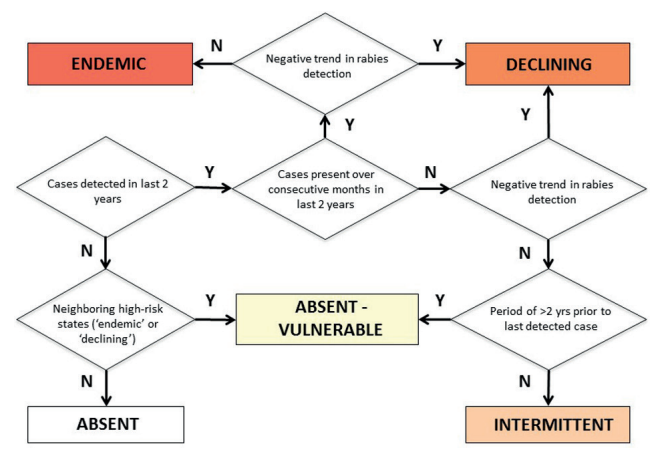

Decision-tree framework.

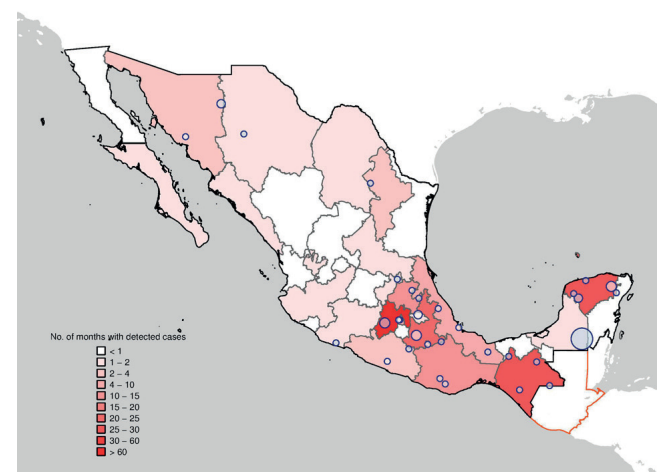

Rabies incursions in Mexico, 2005-2015. Blue circles indicate incursion locations, and resulting outbreak sizes, with darker shading for more recent incursions. Red shading indicates the duration of endemic circulation over the ten-year period.

\section{Keywords}

Rabies elimination; Incursion detection; Policy guidelines; Programme management

\section{Acknowledgments}

This research was supported by the Wellcome Trust. Data were provided by the PAHO and regional government and stakeholders in Mexico.

\section{References}

1. OIE. Global elimination of dog-mediated human rabies-The time is now! Report of the Rabies Global Conference. Geneva, Switzerland, 2015.

2. Vigilato MAN, Clavijo A, Knobl T, Silva HMT, Cosivi O, Schneider MC, Leanes LF, Belotto AJ, Espinal MA. Progress towards eliminating canine rabies: policies and perspectives from Latin America and the Caribbean. Phil Trans R Soc B, 2013; 368.

\section{*Kristyna Rysava}

E-mail: istyrysava@gmail.com 